\title{
Molecular identification and interaction assay of the gene (OsUbc13) encoding a ubiquitin-conjugating enzyme in rice
}

\author{
Ya WANG ${ }^{1,2}$, Meng-yun XU ${ }^{1}$, Jian-ping LIU ${ }^{1}$, Mu-gui $\mathrm{WANG}^{1}$, Hai-qing $\mathrm{YIN}^{2}$, Ju-min $\mathrm{TU}^{\dagger 11}$ \\ ('Institute of Crop Science, College of Agriculture and Biotechnology, Zhejiang University, Hangzhou 310058, China) \\ ( ${ }^{2}$ Cereal Crops Institute, Henan Academy of Agricultural Sciences, Zhengzhou 450002, China) \\ "E-mail: jtu@zju.edu.cn
}

Received Oct. 24, 2013; Revision accepted May 13, 2014; Crosschecked June 24, 2014

\begin{abstract}
The ubiquitin (Ub)-conjugating enzyme, Ubc13, has been known to be involved in error-free DNA damage tolerance (or post-replication repair) via catalyzing Lys63-linked polyubiquitin chains formation together with a Ubc variant. However, its functions remain largely unknown in plant species, especially in monocotyledons. In this study, we cloned a Ub-conjugating enzyme, OsUbc13, that shares the conserved domain of Ubc with AtUBC13B in Oryza sativa L., which encodes a protein of 153 amino acids; the deduced sequence shares high similarities with other homologs. Real-time quantitative polymerase chain reaction (PCR) indicated that OsUbc13 transcripts could be detected in all tissues examined, and the expression level was higher in palea, pistil, stamen, and leaf, and lower in root, stem, and lemma; the expression of OsUbc13 was induced by low temperature, methylmethane sulfate (MMS), and $\mathrm{H}_{2} \mathrm{O}_{2}$, but repressed by mannitol, abscisic acid (ABA), and $\mathrm{NaCl}$. OsUbc13 was probably localized in the plasma and nuclear membranes. About 20 proteins, which are responsible for the positive yeast two-hybrid interaction of OsUbc13, were identified. These include the confirmed OsVDAC (correlated with apoptosis), OsMADS1 (important for development of floral organs), OsB22EL8 (related to reactive oxygen species (ROS) scavenging and DNA protection), and OsCROC-1 (required for formation of Lys63 polyubiquitylation and error-free DNA damage tolerance). The molecular characterization provides a foundation for the functional study of OsUbc13.
\end{abstract}

Key words: Ubc13, DNA damage tolerance, Oryza sativa, Real-time quantitative PCR, Yeast two-hybrid doi: $10.1631 /$ jzus.B1300273

Document code: A

CLC number: Q785

\section{Introduction}

Ubiquitin ( $\mathrm{Ub}$ ) is a highly conserved 76-residue protein, which can be covalently joined to the lysine (K) residues of target proteins as a single $\mathrm{Ub}$ or poly-Ub chain in eukaryotes (Jentsch, 1992; Grisvard et al., 2010). Protein ubiquitination is an essential post-translational modification system that serves as a signaling mechanism in diverse cellular events, including ribosome biogenesis (Finley et al., 1989), DNA repair (Jentsch et al., 1987; Hofmann and

\footnotetext{
Corresponding author

* Project supported by the National High-Tech R\&D Program (863) of China (No. 2006AA10Z159) and the National Natural Science Foundation of China (No. 30871502)

(C) Zhejiang University and Springer-Verlag Berlin Heidelberg 2014
}

Pickart, 1999; Pastushok and Xiao, 2004), protein endocytosis (Galan and Haguenauer-Tsapis, 1997), cell-cycle control (Wei et al., 2004), nuclear factor kappa B (NF-kB)-dependent signal transduction (Chen et al., 1996; Tokunaga et al., 2009), and other cellular processes (Finley et al., 1987). Ubiquitination is accomplished by a series of three enzymatic systems including Ub-activating enzyme (E1), Ub-conjugating enzyme (Ubc or E2), and Ub ligase (E3). In addition, $\mathrm{Ub}$ molecules can be linked to each other through an isopeptide bond formed between the C-terminal Gly76 of a donor and one of seven lysine residues of an acceptor (McKenna et al., 2003), leading to the formation of poly-Ub chains (Chau et al., 1989). Conventional poly-Ub via Gly76-Lys48 is the principal signal for proteolysis through $26 \mathrm{~S}$ proteasomes 
(Hochstrasser, 1996), while Gly76-Lys63 poly-Ub regulates diverse activities in a non-proteolytic way (Pickart, 2001). So far, Ubc13 is the only known Ubc capable of catalyzing the Lys63-linked polyubiquitylation reaction and this function requires interaction with the Ubc variant (Uev, which shares a similar sequence and structure with the E2 Ub-conjugating enzymes, but lacks the active cysteine residue); thus, it is unlike other Ubc proteins that catalyze a conventional Lys48-linked polyubiquitylation reaction (Hofmann and Pickart, 1999; McKenna et al., 2001).

Ubc13 was originally identified as bendless because its loss-of-function mutation affected neuronal synaptic connectivity in Drosophila melanogaster (Muralidhar and Thomas, 1993; Oh et al., 1994). The yeast $u b c 13$ null mutant displays sensitivity to DNA-damaging agents such as ultraviolet (UV) or methylmethane sulfate (MMS) and a high spontaneous mutation rate (Brusky et al., 2000). Lys63-linked chains catalyzed by the Ubc13-Uev (Mms2) complex are required for error-free DNA damage tolerance (DDT, also known as post-replication repair (PRR)) by polyubiquitinating the proliferating cell nuclear antigen (PCNA) (Broomfield et al., 1998; 2001; Hofmann and Pickart, 1999; Hoege et al., 2002) in Saccharomyces cerevisiae. The human Ubc13 protein (hUbc13) has also been cloned (Yamaguchi et al., 1996). The transcription factor NF-kB, which is sequestered in the cytoplasm via interacting with an inhibitory protein of the I $\kappa \mathrm{B}$ family, controls many processes including immunity, inflammation, and apoptosis. IкB proteins can be rapidly phosphorylated by an IкB kinase (IKK) complex and then degraded (Weil and Israel, 2004; Chen, 2005). hUbc13-Uev1A complex is involved in activating the tumor necrosis factor receptor-associated factor 6 (TRAF6)-mediated IKK by catalyzing the synthesis of Lys63-linked poly-Ub chains, which perform polyubiquitination of TRAF6 (Chen, 2012). Mouse Ubc13 is able to functionally complement the budding yeast $u b c 13$ null mutation (Ashley et al., 2002). Ubc13 appears to be essential for the thymocyte $\mathrm{T}$ cell receptor (TCR)mediated NF- $\mathrm{KB}$ activation at the early time points and transforming growth factor- $\beta$-activated kinase 1 (TAK1) phosphorylation in innate immune cells of mice (Sato et al., 2005; Yamamoto et al., 2006b). The deletion of the Ubc13 in mice results in severe loss of blood cells together with atrophy of the thymus and bone marrow, showing that Ubc13 also has a pivotal role in regulating hematopoiesis (Wu et al., 2009). In both zebrafish and Arabidopsis thaliana, there are two $U B C 13$ genes, $u b c 13 a$ and $u b c 13 b$ or $A t U B C 13 A$ and $A t U B C 13 B$. All these four Ubc13 proteins can physically interact with human or yeast Mms 2 and are able to functionally complement the yeast $u b c 13$ null mutant for spontaneous mutagenesis and sensitivity to DNA-damaging agents (Wen et al., 2006; Li et al., 2010); this implies the existence of a Lys63-linked polyubiquitylation reaction and error-free DDT pathway in zebrafish and A. thaliana. ShUbc13 is also capable of physically interacting with ShUev and is probably involved in DNA damage response in the ciliate Sterkiella histriomuscorum (Grisvard et al., 2010). Recently, studies of the Ubcl3 gene in rice have been carried out. OsUbc13 showed ubiquitous expression at a high level even under biotic or abiotic stresses, and its expression can complement the errorfree PRR defects of the yeast $u b c 13$ null mutant. Moreover, OsUbc13 physically interacts with both Mms2 and Uev1A, and catalyzes K63-linked polyubiquitination in vitro (Zang et al., 2012).

Rice is a prominent model for monocotyledonous plants and one of the most important food crops (Yang et al., 2011). However, only limited information is available on Ubc13 in plant species. In this study, we cloned and analyzed the molecular characterization of $O s U b c 13$, and studied its subcellular localization and interacting proteins, providing a foundation for further investigating its function and molecular mechanism.

\section{Materials and methods}

\subsection{Plant materials and treatments}

The mature seeds of a rice variety, Nipponbare, were dehulled and sterilized in $10 \%(0.1 \mathrm{~g} / \mathrm{ml}) \mathrm{NaClO}$ for $15 \mathrm{~min}$, germinated and grown in semi-solid $1 / 2$ MS medium (Murashige and Skoog, 1962) at $28{ }^{\circ} \mathrm{C}$ with a photoperiod of $16 \mathrm{~h} \mathrm{light} / 8 \mathrm{~h}$ dark for two weeks. Seedlings were transferred to Yoshida solution (Yoshida et al., 1976) for two weeks, and finally transferred outside and grown naturally in a field. Plant components at different developmental stages (roots and leaves from the four-week-old seedlings; stem, stamen, pistil, lemma, and palea from the panicles 
at $15 \mathrm{~d}$ after flowering) were collected for real-time polymerase chain reaction (PCR).

To analyze the expression in response to hormone and abiotic stresses, an embryonic cell culture line from the japonica line Nipponbare under culture conditions of $28{ }^{\circ} \mathrm{C}$ in the darkroom was used. For low-temperature treatment, the embryonic cell cultures were directly transferred to a $4{ }^{\circ} \mathrm{C}$ incubator from the normal growth conditions. The culture at $28^{\circ} \mathrm{C}$ in the darkroom was employed as the control. For mannitol, $\mathrm{H}_{2} \mathrm{O}_{2}$, MMS, abscisic acid (ABA), and $\mathrm{NaCl}$ treatments, the embryonic cell cultures were transferred from semi-solid N6 medium to the liquid N6 medium supplemented with $300 \mathrm{mmol} / \mathrm{L}$ mannitol, $20 \mathrm{mmol} / \mathrm{L} \mathrm{H}_{2} \mathrm{O}_{2}, 0.01 \% \mathrm{MMS}, 100 \mu \mathrm{mol} / \mathrm{L} \mathrm{ABA}$, or $200 \mathrm{mmol} / \mathrm{L} \mathrm{NaCl}$, respectively. The embryonic cell cultures grown in the same liquid N6 medium without supplementary components were used as controls. All the cultures, except for the control at $0 \mathrm{~h}$, were placed in a shaker at $200 \mathrm{r} / \mathrm{min}$ at $28^{\circ} \mathrm{C}$ and sampled at $2,4,8$, 12 , and $24 \mathrm{~h}$. Each treatment was repeated three times.

All materials were quickly frozen in liquid nitrogen and then stored at $-80^{\circ} \mathrm{C}$ for RNA extraction.

\subsection{Identification and cloning of OsUbc13 gene}

OsUbc13, corresponding to protein spot 5002, was identified from the specific-organ-inducing medium-induced somatic root and shoot regeneration system in our previous study (data not shown). Analysis of resultant peptides obtained from mass spectrum assay was performed by Expasy (http:// prosite.expasy.org/; http://web.expasy.org/compute_pi/).

The full length complementary DNA (cDNA) of OsUbcl 3 gene was amplified by reverse transcription PCR (RT-PCR). The gene-specific primers for RT-PCR were Ubc13-F (5'-GAATTCATGGCCAAC AGCAACCTCC-3') and Ubc13-R (5'-CCCGGGTT ATGCACCGCTGGCATACA-3') with EcoRI and SmaI restriction sites, respectively. Purified PCR products were cloned into pMD18-T vector (TaKaRa) and then confirmed by sequencing (Invitrogen). The resulting plasmid was called pMDUbc13.

\subsection{Phylogenic analysis of Ubc13 proteins}

For analysis of amino acid sequence identity, DNAMAN software was used. Amino acid sequences of Ubc13 from different species were aligned using ClustalW2. Analysis of phylogeny was performed using MEGA4 via the neighbor-joining method (Tamura et al., 2007). All the amino acid sequences (given in Online Resource 1) were retrieved from the NCBI database (http://www.ncbi.nlm.nih.gov/).

\subsection{Expression analysis of $\mathrm{OSUbc13}$ gene by real- time PCR}

Total RNA was isolated from rice materials using Trizol Reagent (Invitrogen, USA) according to the manufacturer's protocol. The first-strand cDNAs were synthesized using the Moloney murine leukemia virus (M-MLV) first-strand synthesis system with $1 \mu \mathrm{g}$ of freshly extracted RNA (Promega). The SYBR Premix Ex Taq Kit (TaKaRa) was used for real-time PCR analysis, with the primers qUbc13-F (5'-ATGG CCAACAGCAACCTCC-3') and qUbc13-R (5'-TTA TGCACCGCTGGCATACA-3'), qActin-F (5'-GACT CTGGTGATGGTGTCAGC-3') and qActin-R (5'-G GCTGGAAGAGGACCTCAGG-3'). The PCR program and the calculation method of relative expression levels of $O s U b c 13$ in different tissues or under different conditions were as previously described (Wang Y. et al., 2012), with the rice Actin I gene as a reference. Here, $\Delta \Delta C_{\mathrm{T}}=\left(C_{\mathrm{T}(\text { Ubc13, test })}-C_{\mathrm{T}(\text { Actin I, test })}\right)^{-}$ $\left(C_{\mathrm{T}(U b c 13 \text {, calibrator })}-C_{\mathrm{T}(A c t i n I \text {, calibrator })}\right)$, where the $C_{\mathrm{T}}$ (cycle threshold) values for both target and reference genes are the means of the triplicate independent PCRs. Here, the roots or embryonic cell cultures without treatment at $0 \mathrm{~h}$ were set as calibrators.

\subsection{Subcellular localization of OsUbc13}

The subcellular localization of OsUbc13 was analyzed by constructing a fusion protein, in which the OsUbc13 coding sequence (CDS) was fused to green fluorescent protein (GFP). The OsUbcl3 CDS was amplified from pMDUbc13 with the primers slUbc13-F (5'-ATGGCCAACAGCAACCTC-3') and slUbc13-R (5'-TTATGCACCGCTGGCATAC-3') and then inserted into the pGWC, which had been digested by Eam1105I. The fragment was then cloned into the pMDC43 vector via LR reaction (Gateway Technology, Catalog Nos. 12535-019 and 12535-027) to be fused to the downstream of GFP, resulting in pMDC43-Ubc13, denominated as pGFP:Ubc13 (given in Online Resource 2). This final construct was transformed in cultured tobacco BY-2 protoplasts using a procedure as reported previously (Sheen, 2001; Lee et al., 2008; Silva et al., 2010) with some 
modifications. Briefly, the suspended BY-2 protoplasts were subcultured after every 3-4 d by transferring $10 \mathrm{ml}$ cells into $40 \mathrm{ml}$ of fresh liquid MS medium. These suspended cells were then transferred to $50 \mathrm{ml}$ conical tubes in a laminar flow hood and were centrifuged at about $500 \times \mathrm{g}$ for $5 \mathrm{~min}$ at $25^{\circ} \mathrm{C}$. After removing the supernatant, the sediment was dissolved in $25 \mathrm{ml}$ of enzyme solution $(1 \%(0.01 \mathrm{~g} / \mathrm{ml})$ cellulase $0.1 \%(1 \mathrm{~g} / \mathrm{L})$ pectinase $\mathrm{Y} 23$, and $0.2 \%(2 \mathrm{~g} / \mathrm{L})$ driselase in $4.3 \mathrm{~g} / \mathrm{L} \mathrm{MS}$ salts, $0.4 \mathrm{~mol} / \mathrm{L}$ sucrose, $0.5 \mathrm{~g} / \mathrm{L}$ 2-morpholinoethanesulfonic acid (MES) hydrate, $0.25 \mathrm{~g} / \mathrm{L} \mathrm{NH} \mathrm{NHO}_{3}, 0.75 \mathrm{~g} / \mathrm{L} \mathrm{CaCl}_{2} \cdot 2 \mathrm{H}_{2} \mathrm{O}$, $\mathrm{pH}$ 5.7) and incubated in a shaker at $200 \mathrm{r} / \mathrm{min}$ at room temperature for $4-5 \mathrm{~h}$. Then the mixture was centrifuged using a swinging bucket rotor at $80 \times g$ for $15 \mathrm{~min}$. The floating protoplast layer was transferred to a new tube and centrifuged for $5 \mathrm{~min}$ at $100 \times \mathrm{g}$ to collect BY-2 protoplasts. Finally, the transformed protoplasts were examined by confocal laser scanning microscopy (Zeiss LSM700).

\subsection{Interaction assay of OsUbc13 by yeast two- hybrid}

Matchmaker Two-Hybrid System 3 (Clontech, http://www.clontech.com/), in which a bait gene is fused to the GAL4 DNA-binding domain while cDNA is fused to the GAL4 activation domain-AD (Fields and Song, 1989; Chien et al., 1991), was used for protein interaction analysis. Briefly, the fragment that was obtained from pMDBTF3 was digested with EcoRI and SmaI was inserted into the pGBKT7 vector to generate construct pGUbc13 (given in Online Resource 2). An AD fusion library of rice calli in pGADT7 (pGADT7-library) was constructed in Clontech. The following test and re-test of positive two-hybrid interaction were performed as previously described (Wang Y. et al., 2012).

\section{Results}

\subsection{Sequence analysis of OsUbc13 protein}

We identified a protein OsUbc13 corresponding to protein spot 5002 in $2 \mathrm{D}$ gels to be associated with somatic root and shoot regeneration in our previous study (data not shown). The complete coding sequence of $O s U b c 13$ contains eight exons and seven introns, and encodes a UBC homolog of 153 amino acids. As Zang et al. (2012) proposed, OsUbc13 (Os01g0673600) is the only Ubcl3 gene in rice because only this gene with a high degree of sequence identity was found using Arabidopsis Ubcl3 genes to BLAST the rice genomic database on NCBI. OsUbc13 shares high similarities with other homologs, ranging from $66.23 \%$ to $98.04 \%$. Comparison of amino acid sequence revealed that all Ubc13s harbor the active site Cys- 88 within the highly conserved UBC domain (Fig. 1a), indicating that UBC13 orthologs are derived from the same origin evolutionarily. Phylogenetic analysis (Fig. 1b) indicated that OsUbc13 is the most closely related to AtUBC13B, followed by AtUBC13A and animal $\mathrm{Ubc} 13 \mathrm{~s}$, and distant from yeast $\mathrm{ScUbc} 13$. This finding is consistent with the previous report declaring that all except two $O s U b c 13$ introns are different from that of $A t U B C 13 B$ in the gene structure while there are obvious differences between $O s U b c 13$ and other Ubc13s (Zang et al., 2012).

\subsection{Expression of $O s U b c 13$ in various rice tissues}

To investigate the expression pattern of OsUbc13, the relative abundance of mRNA in different tissues including root, stem, leaf, stamen, pistil, lemma, and palea was quantified. The results revealed that the endogenous $\mathrm{Os}_{\mathrm{S}} \mathrm{Ub} 13$ was constitutively expressed in all tissues examined and the abundance level was highest in palea, followed by pistil, leaf, stamen, root, and stem, and lowest in lemma (Fig. 2). These results implied that the OsUbc13 is important for both vegetative growth and reproductive development in rice, especially that of palea, pistil, and leaf.

\subsection{Expression of $\mathrm{OsUbc13}$ in different stress conditions}

In plants, many gene transcripts are very unstable, which is important for the tight control of their temporal and spatial expression patterns (Lee et al., 2006). In order to determine the expression of OsUbcl3 in response to environmental conditions, its transcript level was analyzed by real-time PCR after treatment with low temperature $\left(4^{\circ} \mathrm{C}\right)$, MMS $(0.01 \%)$, $\mathrm{H}_{2} \mathrm{O}_{2}(20 \mathrm{mmol} / \mathrm{L})$, osmotic pressure $(300 \mathrm{mmol} / \mathrm{L}$ mannitol), ABA $(100 \mu \mathrm{mol} / \mathrm{L})$, and $\mathrm{NaCl}(200 \mathrm{mmol} / \mathrm{L})$. The results showed that the expression of $\mathrm{OsUbc13}$ 
(a)
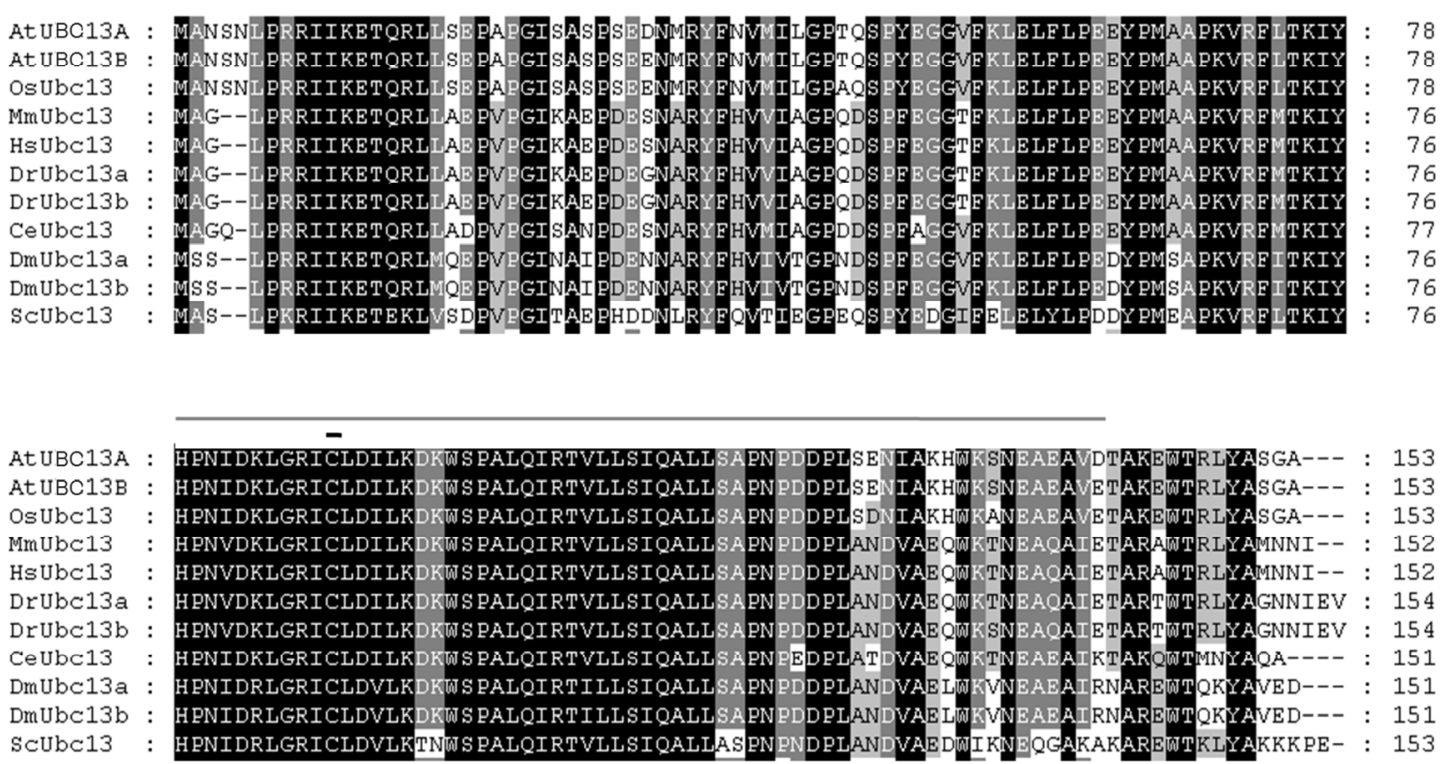

(b)



Fig. 1 Amino acid sequence alignment (a) and phylogenic analysis (b) of Ubc13s from different species The gray and single overline in (a) indicates the conserved UBC domain of Ubc13s; the dark and single overline in (a) indicates the conserved cysteine residue of Ubc13s. Bootstrap values are shown at each node in (b). AtUBC13A: AEE36165; AtUBC13B: AEE29516.1; OsUbc13: NP_001043834.1; MmUbc13: AAK74128.1; HsUbc13: BAA11675.1; DrUbc13a: AAI64904.1; DrUbc13b: AAH53141.1; CeUbc13: NP 500272.2; DmUbc13a: AAF48338.1; DmUbc13b: ACZ95287.1; ScUbc13: CAA67806.1



Fig. 2 Expression analysis of $O s U b c 13$ in various rice tissues

The expression of $O s U b c 13$ in root was set as 1 . Data were expressed as mean \pm standard deviation (SD) of triplicate measurements was induced by low temperature $\left(4^{\circ} \mathrm{C}\right), 0.01 \% \mathrm{MMS}$, and $20 \mathrm{mmol} / \mathrm{L} \mathrm{H}_{2} \mathrm{O}_{2}$ (Figs. 3a-3c), but repressed by $300 \mathrm{mmol} / \mathrm{L}$ mannitol, $100 \mu \mathrm{mol} / \mathrm{L} \mathrm{ABA}$, and $200 \mathrm{mmol} / \mathrm{L} \mathrm{NaCl}$ (Figs. 3d-3f). For low temperature treatment, around four-fold transcripts in the treated samples were observed, compared with the controls at $2 \mathrm{~h}$, the difference reached its maximum value at $12 \mathrm{~h}$, but the level of expression decreased although it was still higher than that of the control at $24 \mathrm{~h}$ (Fig. 3a). For MMS and $\mathrm{H}_{2} \mathrm{O}_{2}$ treatments, the maximum level of expression was at 4 and $8 \mathrm{~h}$, respectively. Moreover, the difference between treated samples and control 

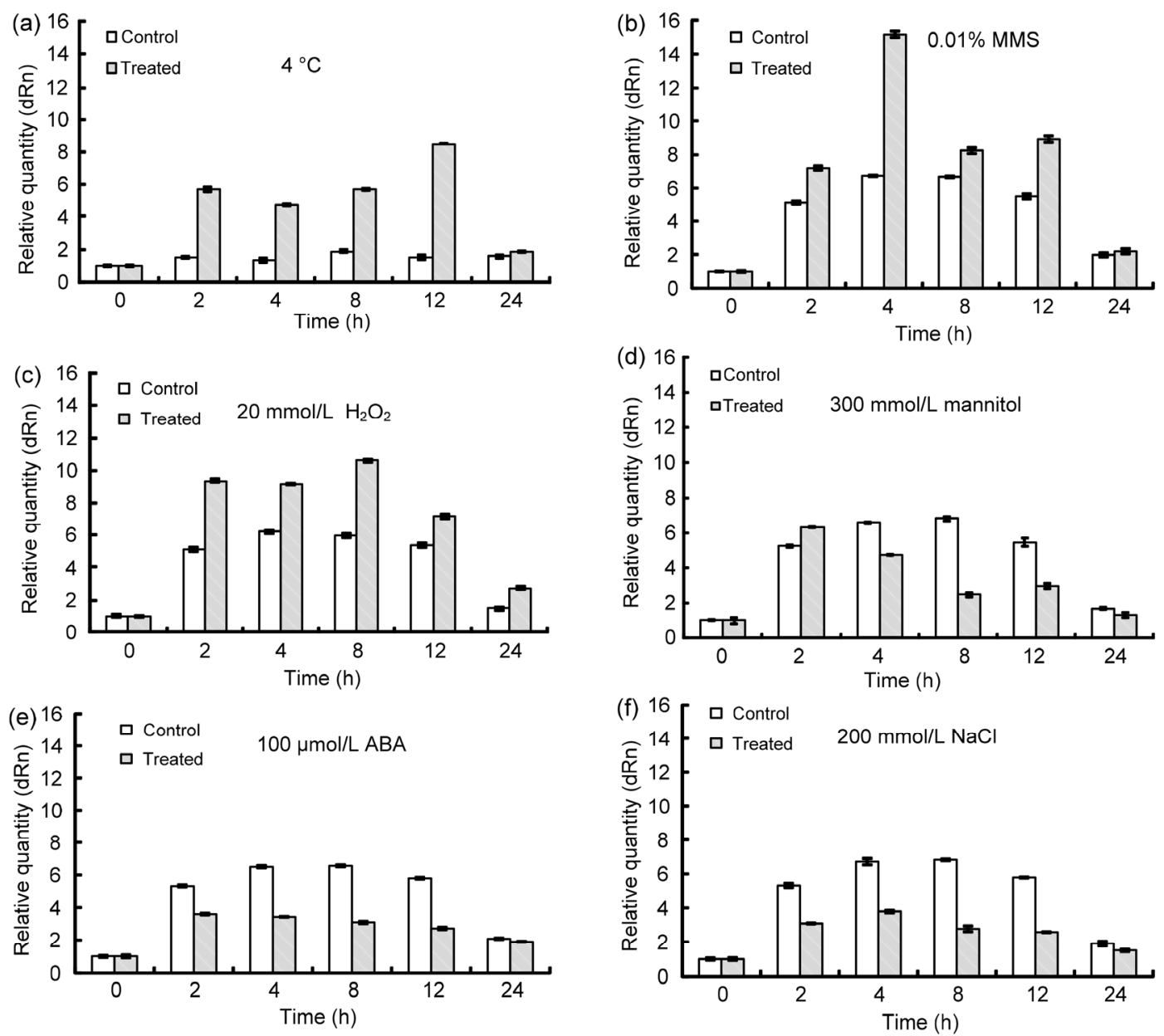

Fig. 3 Expression pattern of $\mathrm{Os} U b c 13$ in response to low temperature of $4{ }^{\circ} \mathrm{C}(\mathrm{a}), 0.01 \% \mathrm{MMS}(\mathrm{b}), 20 \mathrm{mmol} / \mathrm{L} \mathrm{H}_{2} \mathrm{O}_{2}$ (c), $300 \mathrm{mmol} / \mathrm{L}$ mannitol (d), $100 \mu \mathrm{mol} / \mathrm{L} \mathrm{ABA} \mathrm{(e),} \mathrm{and} 200 \mathrm{mmol} / \mathrm{L} \mathrm{NaCl}$ (f)

The detection was performed three times and the data were presented as mean \pm standard deviation (SD). The expression of Os Ubcl3 of control at $0 \mathrm{~h}$ was set as 1

was smaller in the case of MMS and $\mathrm{H}_{2} \mathrm{O}_{2}$ treatments (Figs. 3b-3c) than in low-temperature treatment (Fig. 3a). For the mannitol treatment, the transcripts were less in the samples treated than in the controls at all time points except at $2 \mathrm{~h}$ (Fig. 3d). The expression patterns of $\mathrm{Os} U b c 13$ in response to $\mathrm{ABA}$ and $\mathrm{NaCl}$ were quite similar; however, the time point at which the maximum expression level occurred was different under different treatments. The former (under ABA treatment) was at $2 \mathrm{~h}$ and the latter (under $\mathrm{NaCl}$ treatment) was at $4 \mathrm{~h}$ (Figs. 3e-3f). Interestingly, the transcripts in the control cultures in liquid N6 medium (Figs. 3b-3f) fluctuated noticeably, compared with those cultured in solid N6 medium (Fig. 3a), indicating that culture conditions may affect the expression level of $O s U b c 13$.

\subsection{Localization of OsUbc13 in the plasma and nuclear membranes}

To determine the subcellular localization of OsUbc13, the fusion construct pMDC43-Ubc13 containing GFP:OsUbc13 CDS and driven by $35 \mathrm{~S}$ promoter was introduced into the protoplasts of cultured Nicotiana benthamiana via polyethylene glycol (PEG)-mediated transformation. The non-fusion construct pMDC43 was used as a control. Here, the tobacco cell system as a model system was selected to study subcellular localization of OsUbc13 for its potential advantages over other cell cultures such as highly synchronized growth rate, high transformation efficiency, and easy monitoring and analysis (Geelen and Inzé, 2001; Tripathi et al., 2013). The data revealed that OsUbc13 was probably localized in the plasma 

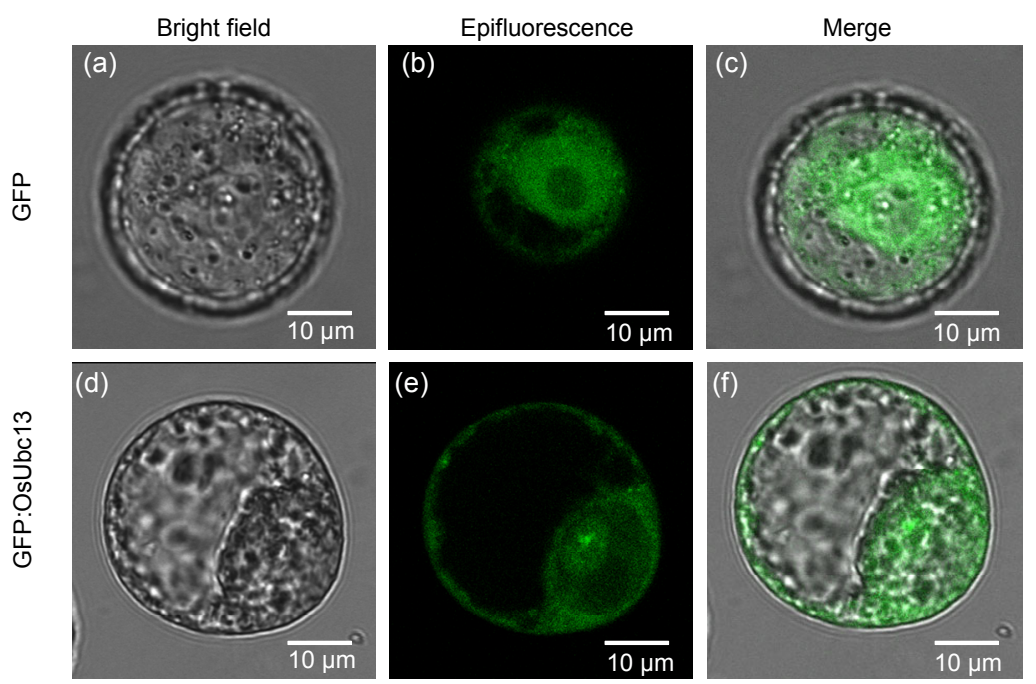

Fig. 4 Subcellular localization of control GFP (a-c) and GFP:OsUbc13 (d-f) fusion proteins transiently expressed in the cultured BY-2 protoplast

GFP:OsUbc13 was localized in the plasma and nuclear membranes. Images were taken in bright field (a, d) for the cell outline and in dark field for green fluorescence (b, e). Images (c) and (f) are superimposed images between (a) and (b) as well as (d) and (e), respectively (Note: for interpretation of the references to color in this figure legend, the reader is referred to the web version of this article)

and nuclear membranes since the fluorescence signal of GFP:OsUbc13 was relatively intense in these areas (Figs. 4d-4f), while control GFP was localized in both the cytoplasm and nucleus (Figs. $4 \mathrm{a}-4 \mathrm{c}$ ). The subcellular localization of OsUbc13 implied that OsUbc13 may function in the membrane system.

\subsection{Potential OsUbc13-interacting partners in yeast two-hybrid assay}

To provide insights into biological function and regulatory mechanism, yeast two-hybrid was performed to determine potential candidates. As the results showed, 81 colonies were positive (Fig. 5a). Out of them, 20 proteins (Table 1), which are responsible for the positive two-hybrid interaction, were identified via PCR colony-screening and DNA sequencing. The protein-protein interactions were verified by co-transformation in yeast AH109 with four genes (out of 20) as representatives. The four genes are OsVDAC (Godbole et al., 2003), OsCROC-1 (Hofmann and Pickart, 1999), OsB22EL8 (Yuan J. et al., 2008), and OsMADS1 (Prasad et al., 2005) of which the function had been defined (Fig. 5b).

Another eight potential OsUbc13-interacting proteins (Table 1), along with these four confirmed genes, are associated with apoptosis (Yang et al., 1997; Kim et al., 2009; Hashimoto et al., 2012; Wang W.M. et al., 2012), DNA repair and damage tolerance



Fig. 5 Yeast two-hybrid analysis with OsUbc13 as the bait (a) The positive colonies on SD/-Ade/-His/-Leu/-Trp/X- $\alpha$-gal plate with OsUbc13 as the bait. (b) The interactions were verified by co-transformation represented by OsB22EL8 (2), OsMADS1 (4), OsCROC-1 (6), and OsVDAC (8). 1, 3, 5, and 7 represent four negative controls of $2,4,6$, and 8 , respectively 
Table 1 List of proteins interacted with OsUbc13 from yeast two-hybrid

\begin{tabular}{lll}
\hline \multicolumn{1}{c}{ Function } & \multicolumn{1}{c}{ Protein description } & GenBank accession No. \\
\hline Apoptosis & Outer mitochondrial membrane protein porin (OsVDAC) & Os09g0361400 \\
& Hypersensitive response (HR)-like lesion-inducer family protein & Os10g0445500 \\
& Similar to cysteine protease inhibitor & Os05g0409300 \\
DNA damage repair & Polyubiquitin & Os06g0681400 \\
& Similar to CROC-1-like protein (OsCROC-1) & Os12g0605400 \\
& Similar to polyubiquitin & Os04g0628100 \\
& Similar to B22EL8 protein (OsB22EL8) & Os05g0111300 \\
& RNA polymerase subunit, RPB5/RPB6-like domain containing protein & Os06g0618600 \\
& Similar to CROC-1-like protein & Os04g0684800 \\
Flower development & Box protein (MADS box protein MADS1) (OsMADS1) & Os03g0215400 \\
& Arf GTPase activating protein family protein & Os01g0951100 \\
& Oryza sativa SKP1 ( -phase kinase-associated protein 1)-like protein 1 & Os05g0530500 \\
Immunological & Pollen allergen Lol p2 family protein & Os04g0317500 \\
responses & Pollen allergen Lol p2 family protein & Os06g0655200 \\
Catabolism & Similar to 3-methylcrotonyl CoA carboxylase biotin-containing subunit & Os12g0605800 \\
Splicing and cell & Similar to U5 small nuclear ribonucleoprotein 200 kDa helicase & Os02g0107000 \\
cycle regulation & Similar to coatomer delta subunit ( $\delta$-coat protein $(\delta$-COP)) & \\
Other & Similar to YyaF/YCHF TRANSFAC/OBG family small GTpase plus & Os08g0199300 \\
& RNA binding domain TGS & Os11g0282300 \\
& Protein of unknown function DUF1685 family protein & Os03g0411500 \\
\hline
\end{tabular}

(Parusel et al., 2006), and floral organ development (Dai et al., 2002; Takacs et al., 2012). The remaining eight proteins (Table 1) are involved in immunological responses (Petersen et al., 2006; Schweimer et al., 2008), catabolism (Song et al., 1994; Weaver et al., 1995; Guan et al., 1999; Obata et al., 2001), splicing and cell-cycle regulation (Lauber et al., 1996; Ehsani et al., 2013). These results indicated that OsUbc13 might function in diverse biological processes.

\section{Discussion}

UBC13 is a Ub-conjugating enzyme involved in error-free DDT (or PRR) by catalyzing the synthesis of Lys63-linked poly-Ub chains together with a Uev (Broomfield et al., 1998) and a variety of stresses (Hiraishi et al., 2006). In this study, we cloned and characterized $O_{s} U b c 13$, which encodes a Ubconjugating protein. Although the amino acid sequence comparison of rice Ubc13 with Ubc13s from other species (Fig. 1a) is similar to previous results (Zang et al., 2012), the range of UBC13 orthologs is wider and all Ubc13s harbor the active site Cys-88 within the highly conserved UBC domain; this indicates that UBC13 orthologs from entire eukaryotic organisms are derived from the same origin evolutionarily. $O s U b c 13$ was constitutively expressed in all the tissues and organs examined (Fig. 2), implying that OsUbc13 may exert important roles in the growth and development of rice. It is worth considering that both $A t U B C 13 s$ and $O s U b c 13$ were not only expressed ubiquitously in different tissues but also kept at a relatively stable transcript level in the previous report (Wen et al., 2006; Zang et al., 2012), and this is a little different from our results. Our data showed that $O s U b c 13$ was expressed abundantly in palea, pistil, leaf, and stamen, compared with its lower expression level in root, stem, and lemma (Fig. 2). In our opinion, the differences, if any, are at least partly due to different research methods (e.g., the detection technology or the method of data analysis). A crossdatabase search on the Genevestigator (https://www. genevestigator.com/gv/doc/plant/introduction.jsp) was performed to investigate the expression profiles of OsUbc13 in the previous report while our results were obtained via real-time PCR using three independent sets of biological samples. 
Previous reports showed that the disruption of the $U b c 13$ gene results in an increased killing by MMS and UV irradiation as well as an increased spontaneous mutation rate in yeast (Brusky et al., 2000), implying that the expression of $U b c 13$ is susceptible to adverse environments, especially those causing damage to DNA. Hence, in order to identify the response of $O s U b c 13$ expression to different stresses, real-time PCR was performed to check the expression pattern of $\mathrm{Os} U b c 13$ under conditions of low temperature $4{ }^{\circ} \mathrm{C}$, mannitol, $\mathrm{H}_{2} \mathrm{O}_{2}$, MMS, ABA, and $\mathrm{NaCl}$. It is reasonable to assume that the expression of $U b c 13$ may be regulated by stresses since Ubc13 is mainly involved in stress responses such as DNA damage. In fact, yeast and zebrafish $U b c 13 s$ are induced by different DNA damaging agents such as MMS (Brusky et al., 2000; Li et al., 2010). Similarly, MMS up-regulated the expression of OsUbc13 (Fig. 3b), and the expression levels under treatments of low temperature and $\mathrm{H}_{2} \mathrm{O}_{2}$ were also higher than those under normal conditions (Figs. 3a and 3c). In contrast, its expression was down-regulated by $\mathrm{ABA}$ and $\mathrm{NaCl}$ (Figs. 3e and 3f). For osmotic pressure treatment, the expression level was increased at $2 \mathrm{~h}$ and after that it decreased continually, compared with the control (Fig. 3d); this was slightly different from the expression pattern of $O s U b c 13$ to other stress treatments. However, the transcript level of $O s U b c 13$ appears to fluctuate under different stresses in this study, and this is in contrast to previous results from Arabidopsis and rice (Wen et al., 2006; Zang et al., 2012). The differences are probably attributable to the experimental methods. Here, we used embryonic cell culture for the analysis of $\mathrm{OsUbcl3}$ gene expression in response to various stresses, and detected its expression at different time points. In conclusion, our data showed that $O s U b c 13$ has an important role in plant responses to stress tolerance and can respond actively to negative circumstances by changing its transcripts. It should be noted that even the "untreated" controls, all of which were cultured in liquid N6 medium, are rather different (Figs. 3b-3f). We think the reason for the differences is probably the different culture conditions. The cultures that had not been placed in the shaker were sampled as controls at $0 \mathrm{~h}$, while controls at the other time points were cultured in the shaker at $200 \mathrm{r} / \mathrm{min}$, implying that culture conditions may affect the gene expression level.
It is important to determine the subcellular localization of corresponding proteins to understand the function of a gene as a whole. In yeast (Ulrich and Jentsch, 2000), mammals (Andersen et al., 2005), and zebrafish (Li et al., 2010), Ubc13 was considered to be a cytoplasmic protein. In the single-celled organism, the ciliate $S$. histriomuscorum, Ubc13 protein shows three main locations: nucleus, cilia, and plasma membrane (Grisvard et al., 2010). However, there has been no report on cellular localization of a plant Ubc13 protein. In this study, we are the first to demonstrate that the fluorescence of GFP:OsUbc13 was more strongly close to the plasma and nuclear membranes (Fig. 4e), implying that OsUbc13 may function in the membrane system; this is a difference from the previous results from yeast, animals, and $S$. histriomuscorum (Ulrich and Jentsch, 2000; Andersen et al., 2005; Grisvard et al., 2010; Li et al., 2010). For further studies, it will be essential to confirm the localization of OsUbc13 via adding a membranespecific marker.

To understand further the functions of Ubc13mediated protein ubiquitination, it is important to study Ubc13-interacting proteins. About 20 proteins (Table 1), most of which have not been described previously, are identified as potential OsUbc13interacting partners. We selected four genes to confirm the interactions (Fig. 5b) since these genes are representative in the several types of genes listed in Table 1 and their functions have already been studied systematically. For instance, Ub-conjugating E2 enzyme variant (Uev) CROC-1, which was originally identified on the basis of its capacity to activate transcription of c-Fos (Rothofsky and Lin, 1997), lacks a critical cysteine residue essential for the catalytic activity of E2 enzymes. Uev can functionally complement the yeast $m m s 2$ null mutant from killing by DNA-damaging agents, including MMS and UV (Xiao et al., 1998). In A. thaliana, all four Uev1 proteins can interact with AtUBC13 or Ubc13 from yeast or human to form a stable complex. Furthermore, when treated with a DNA-damaging agent, both seeds and pollen of uevld plants germinated poorly (Wen et al., 2008). Zang et al. (2012) reported that OsUbc13 physically interacts with Mms2 and Uev1A, which are hUbc13 partners, and catalyzes K63 polyubiquitination in vitro. We demonstrated here that OsUbc13 physically interacted with OsCROC-1 (Fig. 5b; Table 1), 
which is essential for Ubc13-mediated Lys63 polyubiquitylation (Hofmann and Pickart, 1999; McKenna et al., 2001; Wen et al., 2006); this once again implies the existence of error-free DDT in plants and the critical role of OsUbc13 in tolerance to DNA damage during seed and pollen germination.

Apoptosis plays an important role during development, homeostasis, and in many diseases such as cancer (Steller, 1995). OsVDAC, which is known to be involved in apoptosis, is another confirmed OsUbc13-interacting protein here. VDAC is a mitochondrial outer membrane ion channel and a critical participant in the apoptosis of animal and plant cells (Al Bitar et al., 2003; Godbole et al., 2003). In plants, over-expression of rice VDAC (OsVDAC4) induces apoptosis (Godbole et al., 2011). In animal cells, knocking down $V D A C l$ by small interfering RNA attenuates endostatin (ES)-induced apoptosis, while over-expression of $V D A C l$ enhances the sensitivity of endothelial cells to ES (Yuan S. et al., 2008). The previous study indicated that apoptosis may be induced via transmembrane receptors that belong to the tumor necrosis factor (TNF) receptor family (Susin et al., 1997) and TNF receptor signaling has been closely linked to TAK1, which needs the involvement of Ubc13 in phosphorylation (Yamamoto et al., 2006a). The yeast two-hybrid results showed that OsUbc13 interacted with OsVDAC (Fig. 5b; Table 1), indicating that $\mathrm{OsUbc13}$ might function in apoptosis via TNF receptor signaling.

The third confirmed OsUbc13-interacting protein OsB22EL8 is a kind of metallothionein (MT) protein. MTs play important roles in maintaining homeostasis and detoxification of metals in both plants and animals; they are known as a kind of low molecular mass and cysteine-rich metal-binding protein (Yuan J. et al., 2008). In plants, studies on the responses of MTs to stress conditions, such as drought and reactive oxygen species (ROS), have also been conducted widely (Akashi et al., 2004; Wong et $a l ., 2004)$. It is well known that both abiotic and biotic stresses can induce the increased ROS such as superoxide, hydrogen peroxide, singlet oxygen, and hydroxyl radicals at certain stages of stress exposure. These ROS may cause non-specific oxidation of proteins and membrane lipids or DNA injury (Pastori and Foyer, 2002; Apel and Hirt, 2004). MTs can improve the stress tolerance of plants by participating in a complex ROS scavenging system to minimize the effects of oxidative stress (Yang et al., 2009). We speculate that OsUbc13 might participate in DNA protection by an ROS-scavenging pathway considering the fact that OsUbc13 interacted with OsB22EL8 (Fig. 5b; Table 1).

OsUbc13 is also probably involved in floral organ identity. OsMADS1, a rice MADS-box factor, which is the last OsUbc13-interacting protein identified in our study (Fig. 5b; Table 1), controls both differentiation and proliferation of specific lemma and palea cell types and is an early-acting regulator of inner floral organs (Prasad et al., 2005). Both the gain-of-function and the loss-of-function of OsMADS1 can lead to severe defects in the specification of lemma and palea (Agrawal et al., 2005). Previous studies on Ubc13 focused on error-free DDT pathways and other cellular processes, and our findings that OsUbc13 interacted with OsMADS1 could open a new research area of Ubc13 in plant flower development.

However, we selected only four of the candidate genes to confirm the interactions without re-testing the others such as hypersensitive response (HR)-like lesion-inducer family protein, pollen allergen Lol p2 family protein, cysteine protease inhibitor, RNA polymerase subunit, and Oryza sativa SKP1 ( $S$-phase kinase-associated protein 1)-like protein 1 (OSK1), so it is not clear whether the interactions are valid for the other proteins. Since the regulation network of these important protein-protein interactions is vital for the study of the function of OsUbc13 in depth, the follow-up work we are carrying out will be focused on confirming all the candidate $O s U b c 13$-interacting genes and then the function analysis of the re-tested genes where the function is unknown. In summary, our findings provide a preliminary foundation for understanding the function of OsUbc13.

\section{Acknowledgements}

We are grateful to Dr. Li-yu HUANG (Chinese Academy of Agricultural Sciences) for technical assistance and Dr. Sun-jie LU (Fudan University, Shanghai, China) for helpful suggestions in the discussion.

\section{Compliance with ethics guidelines}

Ya WANG, Meng-yun XU, Jian-ping LIU, Mu-gui WANG, Hai-qing YIN, and Ju-min TU declare that they have no conflict of interest.

This article does not contain any studies with human or animal subjects performed by any of the authors. 


\section{References}

Agrawal, G.K., Abe, K., Yamazaki, M., et al., 2005. Conservation of the E-function for floral organ identity in rice revealed by the analysis of tissue culture-induced loss-of-function mutants of the OSMADS1 gene. Plant Mol. Biol., 59(1):125-135. [doi:10.1007/s11103-0052161-y]

Akashi, K., Nishimura, N., Ishida, Y., et al., 2004. Potent hydroxyl radical-scavenging activity of drought-induced type-2 metallothionein in wild watermelon. Biochem. Biophys. Res. Commun., 323(1):72-78. [doi:10.1016/ j.bbrc.2004.08.056]

Al Bitar, F., Roosens, N., Smeyers, M., et al., 2003. Sequence analysis, transcriptional and posttranscriptional regulation of the rice vdac family. Biochem. Biophys. Acta, 1625(1):43-51.

Andersen, P.L., Zhou, H., Pastushok, L., et al., 2005. Distinct regulation of Ubc13 functions by the two ubiquitinconjugating enzyme variants Mms2 and UEV1A. J. Cell Biol., 170(5):745-755. [doi:10.1083/jcb.200502113]

Apel, K., Hirt, H., 2004. Reactive oxygen species: metabolism, oxidative stress, and signal transduction. Annu. Rev. Plant Biol., 55(1):373-399. [doi:10.1146/annurev.arplant.55. 031903.141701]

Ashley, C., Pastushok, L., McKenna, S., et al., 2002. Roles of mouse UBC13 in DNA postreplication repair and Lys63-linked ubiquitination. Gene, 285(1-2):183-191. [doi:10.1016/S0378-1119(02)00409-2]

Broomfield, S., Chow, B.L., Xiao, W., 1998. MMS2, encoding a ubiquitin-conjugating-enzyme-like protein, is a member of the yeast error-free postreplication repair pathway. PNAS, 95(10):5678-5683. [doi:10.1073/pnas.95.10.5678]

Broomfield, S., Hryciw, T., Xiao, W., 2001. DNA postreplication repair and mutagenesis in Saccharomyces cerevisiae. Mutat. Res., 486(3):167-184. [doi:10.1016/S09218777(01)00091-X]

Brusky, J., Zhu, Y., Xiao, W., 2000. UBC13, a DNA-damageinducible gene, is a member of the error-free postreplication repair pathway in Saccharomyces cerevisiae. Curr. Genet., 37(3):168-174. [doi:10.1007/s002940050515]

Chau, V., Tobias, J.W., Bachmair, A., et al., 1989. A multiubiquitin chain is confined to specific lysine in a targeted short-lived protein. Science, 243(4898):1576-1583. [doi:10.1126/science.2538923]

Chen, Z.J., 2005. Ubiquitin signalling in the NF-кB pathway. Nat. Cell Biol., 7(8):758-765. [doi:10.1038/ncb0805-758]

Chen, Z.J., 2012. Ubiquitination in signaling to and activation of IKK. Immunol. Rev., 246(1):95-106. [doi:10.1111/j. 1600-065X.2012.01108.x]

Chen, Z.J., Parent, L., Maniatis, T., 1996. Site-specific phosphorylation of I $\mathrm{B} \alpha$ by a novel ubiquitination-dependent protein kinase activity. Cell, 84(6):853-862. [doi:10. 1016/S0092-8674(00)81064-8]

Chien, C.T., Bartel, P.L., Sternglanz, R., et al., 1991. The two-hybrid system: a method to identify and clone genes for proteins that interact with a protein of interest. PNAS, 88(21):9578-9582. [doi:10.1073/pnas.88.21.9578]

Dai, L., Xu, L., Huang, D., et al., 2002. ASK1 physically interacts with COI1 and is required for male fertility in Arabidopsis. Sci. China C Life Sci., 45(6):631-636. [doi:10.1007/BF02879751]

Ehsani, A., Alluin, J.V., Rossi, J.J., 2013. Cell cycle abnormalities associated with differential perturbations of the human U5 snRNP associated U5-200kD RNA helicase. PLoS ONE, 8(4):e62125. [doi:10.1371/journal.pone.00 62125]

Fields, S., Song, O., 1989. A novel genetic system to detect protein-protein interactions. Nature, 340(6230):245-246. [doi:10.1038/340245a0]

Finley, D., Ozkaynak, E., Varshavsky, A., 1987. The yeast poly-ubiquitin gene is essential for resistance to high temperatures, starvation and other stresses. Cell, 48(6): 1035-1046. [doi:10.1016/0092-8674(87)90711-2]

Finley, D., Bartel, B., Varshavsky, A., 1989. The tails of ubiquitin precursors are ribosomal proteins whose fusion to ubiquitin facilitates ribosome biogenesis. Nature, 338(6214):394-401. [doi:10.1038/338394a0]

Galan, J.M., Haguenauer-Tsapis, R., 1997. Ubiquitin lys63 is involved in ubiquitination of a yeast plasma membrane protein. EMBO J., 16(19):5847-5854. [doi:10.1093/emboj/ 16.19.5847]

Geelen, D.N., Inzé, D.G., 2001. A bright future for the bright yellow-2 cell culture. Plant Physiol., 127(4):1375-1379. [doi:10.1104/pp.010708]

Godbole, A., Varghese, J., Sarin, A., et al., 2003. VDAC is a conserved element of death pathways in plant and animal systems. Biochim. Biophys. Acta, 1642(1-2):87-96. [doi:10.1016/S0167-4889(03)00102-2]

Godbole, A., Mitra, R., Dubey, A.K., et al., 2011. Bacterial expression, purification and characterization of a rice voltage-dependent, anion-selective channel isoform, OsVDAC4. J. Membr. Biol., 244(2):67-80. [doi:10.1007/ s00232-011-9399-x]

Grisvard, J., Aubusson-Fleury, A., Baroin-Tourancheau, A., 2010. Multiple uses of Lys63-polyubiquitination in the ciliate Sterkiella histriomuscorum. Protist, 161(3):479488. [doi:10.1016/j.protis.2010.01.004]

Guan, X., Diez, T., Prasad, T.K., et al., 1999. Geranoyl-CoA carboxylase: a novel biotin-containing enzyme in plants. Arch. Biochem. Biophys., 362(1):12-21. [doi:10.1006/ abbi.1998.1014]

Hashimoto, M., Komatsu, K., Maejima, K., et al., 2012. Identification of three MAPKKKs forming a linear signaling pathway leading to programmed cell death in Nicotiana benthamiana. BMC Plant Biol., 12(1):103. [doi:10.1186/ 1471-2229-12-103]

Hiraishi, H., Mochizuki, M., Takagi, H., 2006. Enhancement of stress tolerance in Saccharomyces cerevisiae by overexpression of ubiquitin ligase Rsp5 and ubiquitinconjugating enzymes. Biosci. Biotechnol. Biochem., 70(11): 2762-2765. [doi:10.1271/bbb.60250] 
Hochstrasser, M., 1996. Ubiquitin-dependent protein degradation. Annu. Rev. Genet., 30(1):405-439. [doi:10. 1146/annurev.genet.30.1.405]

Hoege, C., Pfander, B., Moldovan, G.L., et al., 2002. RAD6dependent DNA repair is linked to modification of PCNA by ubiquitin and SUMO. Nature, 419(6903):135-141. [doi:10.1038/nature00991]

Hofmann, R.M., Pickart, C.M., 1999. Noncanonical MMS2encoded ubiquitin-conjugating enzyme functions in assembly of novel polyubiquitin chains for DNA repair. Cell, 96(5):645-653. [doi:10.1016/S0092-8674(00)80575-9]

Jentsch, S., 1992. The ubiquitin-conjugation system. Annu. Rev. Genet., 26(1):179-207. [doi:10.1146/annurev.ge.26. 120192.001143]

Jentsch, S., McGrath, J.P., Varshavsky, A., 1987. The yeast DNA repair gene RAD6 encodes a ubiquitin-conjugating enzyme. Nature, 329(6135):131-134. [doi:10.1038/329 131a0]

Kim, J.A., Cho, K., Singh, R., et al., 2009. Rice OsACDR1 (Oryza sativa accelerated cell death and resistance 1) is a potential positive regulator of fungal disease resistance. Mol. Cells, 28(5):431-439. [doi:10.1007/s10059-0090161-5]

Lauber, J., Fabrizio, P., Teigelkamp, S., et al., 1996. The HeLa $200 \mathrm{kDa}$ U5 snRNP-specific protein and its homologue in Saccharomyces cerevisiae are members of the DEXHbox protein family of putative RNA helicases. EMBO J., 15(15):4001-4015.

Lee, B.H., Kapoor, A., Zhu, J., et al., 2006. STABILIZED1, a stress-upregulated nuclear protein, is required for pre-mRNA splicing, mRNA turnover, and stress tolerance in Arabidopsis. Plant Cell, 18(7):1736-1749. [doi:10. 1105/tpc.106.042184]

Lee, L.Y., Fang, M.J., Kuang, L.Y., et al., 2008. Vectors for multi-color bimolecular fluorescence complementation to investigate protein-protein interactions in living plant cells. Plant Methods, 4(1):24. [doi:10.1186/1746-48114-24]

Li, J., Wen, R., Andersen, P., et al., 2010. Zebrafish Ubc13 is required for Lys63-linked polyubiquitination and DNA damage tolerance. Mol. Cell. Biochem., 343(1-2):173-182. [doi:10.1007/s11010-010-0511-9]

McKenna, S., Spyracopoulos, L., Moraes, T., et al., 2001. Noncovalent interaction between ubiquitin and the human DNA repair protein Mms2 is required for Ubc13mediated polyubiquitination. J. Biol. Chem., 276(43): 40120-40126. [doi:10.1074/jbc.M102858200]

McKenna, S., Hu, J., Moraes, T., et al., 2003. Energetics and specificity of interactions within Ub.Uev.Ubc13 human ubiquitin conjugation complexes. Biochemistry, 42(26): 7922-7930. [doi:10.1021/bi034480t]

Muralidhar, M.G., Thomas, J.B., 1993. The Drosophila bendless gene encodes a neural protein related to ubiquitinconjugating enzymes. Neuron, 11(2):253-266. [doi:10. 1016/0896-6273(93)90182-Q]

Murashige, T., Skoog, F., 1962. A revised medium for rapid growth and bioassays with tobacco cultures. Physiol. Plant., 15(3):473-493. [doi:10.1111/j.1399-3054.1962. tb08052.x]

Obata, K., Fukuda, T., Morishita, R., et al., 2001. Human biotin-containing subunit of 3-methylcrotonyl-CoA carboxylase gene (MCCA): cDNA sequence, genomic organization, localization to chromosomal band 3q27, and expression. Genomics, 72(2):145-152. [doi:10.1006/geno. 2000.6366]

Oh, C.E., McMahon, R., Benzer, S., et al., 1994. bendless, a Drosophila gene affecting neuronal connectivity, encodes a ubiquitin-conjugating enzyme homolog. J. Neurosci., 14(5):3166-3179.

Parusel, C.T., Kritikou, E.A., Hengartner, M.O., et al., 2006. URI-1 is required for DNA stability in C. elegans. Development, 133(4):621-629. [doi:10.1242/dev.02235]

Pastori, G.M., Foyer, C.H., 2002. Common components, networks, and pathways of cross-tolerance to stress. The central role of "redox" and abscisic acid-mediated controls. Plant Physiol., 129(2):460-468. [doi:10.1104/pp. 011021]

Pastushok, L., Xiao, W., 2004. DNA postreplication repair modulated by ubiquitination and sumoylation. Adv. Protein Chem., 69:279-306. [doi:10.1016/S0065-3233(04) 69010-3]

Petersen, A., Suck, R., Lindner, B., et al., 2006. Phl p 3: structural and immunological characterization of a major allergen of timothy grass pollen. Clin. Exp. Allergy, 36(6): 840-849. [doi:10.1111/j.1365-2222.2006.02505.x]

Pickart, C.M., 2001. Ubiquitin enters the new millennium. Mol. Cell, 8(3):499-504. [doi:10.1016/S1097-2765(01)00347-1]

Prasad, K., Parameswaran, S., Vijayraghavan, U., 2005. OsMADS1, a rice MADS-box factor, controls differentiation of specific cell types in the lemma and palea and is an early-acting regulator of inner floral organs. Plant J., 43(6):915-928. [doi:10.1111/j.1365-313X.2005.02504.x]

Rothofsky, M.L., Lin, S.L., 1997. CROC-1 encodes a protein which mediates transcriptional activation from the human FOS promoter. Gene, 195(2):141-149. [doi:10.1016/ S0378-1119(97)00097-8]

Sato, S., Sanjo, H., Takeda, K., et al., 2005. Essential function for the kinase TAK1 in innate and adaptive immune responses. Nat. Immunol., 6(11):1087-1095. [doi:10.1038/ ni1255]

Schweimer, K., Petersen, A., Suck, R., et al., 2008. Solution structure of Phl p 3, a major allergen from timothy grass pollen. Biol. Chem., 389(7):919-923. [doi:10.1515/BC. 2008.102]

Sheen, J., 2001. Signal transduction in maize and Arabidopsis mesophyll protoplasts. Plant Physiol., 127(4):1466-1475. [doi:10.1104/pp.010820]

Silva, A.T., Nguyen, A., Ye, C., et al., 2010. Conjugated polymer nanoparticles for effective siRNA delivery to tobacco BY-2 protoplasts. BMC Plant Biol., 10(1):291. [doi:10.1186/1471-2229-10-291]

Song, J., Wurtele, E.S., Nikolau, B.J., 1994. Molecular cloning 
and characterization of the cDNA coding for the biotincontaining subunit of 3-methylcrotonoyl-CoA carboxylase: identification of the biotin carboxylase and biotincarrier domains. PNAS, 91(13):5779-5783. [doi:10.1073/ pnas.91.13.5779]

Steller, H., 1995. Mechanisms and genes of cellular suicide. Science, 267(5203):1445-1449. [doi:10.1126/science.787 8463]

Susin, S.A., Zamzami, N., Castedo, M., et al., 1997. The central executioner of apoptosis: multiple connections between protease activation and mitochondria in Fas/ APO-1/CD95- and ceramide-induced apoptosis. J. Exp. Med., 186(1):25-37. [doi:10.1084/jem.186.1.25]

Takacs, E.M., Suzuki, M., Scanlon, M.J., 2012. Discolored1 (DSC1) is an ADP-ribosylation factor-GTPase activating protein required to maintain differentiation of maize kernel structures. Front. Plant Sci., 3:115. [doi:10.3389/ fpls.2012.00115]

Tamura, K., Dudley, J., Nei, M., et al., 2007. MEGA4: molecular evolutionary genetics analysis (MEGA) software version 4.0. Mol. Biol. Evol., 24(8):1596-1599. [doi:10. 1093/molbev/msm092]

Tokunaga, F., Sakata, S., Saeki, Y., et al., 2009. Involvement of linear polyubiquitylation of NEMO in NF- $\mathrm{kB}$ activation. Nat. Cell Biol., 11(2):123-132. [doi:10.1038/ncb 1821]

Tripathi, P., Rabara, R.C., Lin, J., et al., 2013. GmWRKY53, a water- and salt-inducible soybean gene for rapid dissection of regulatory elements in BY-2 cell culture. Plant Signal. Behav., 8(5):e24097. [doi:10.4161/psb.24097]

Ulrich, H.D., Jentsch, S., 2000. Two RING finger proteins mediate cooperation between ubiquitin-conjugating enzymes in DNA repair. EMBO J., 19(13):3388-3397. [doi:10.1093/emboj/19.13.3388]

Wang, W.M., Ma, X.F., Zhang, Y., et al., 2012. PAPP2C interacts with the atypical disease resistance protein RPW8.2 and negatively regulates salicylic aciddependent defense responses in Arabidopsis. Mol. Plant, 5(5):1125-1137. [doi:10.1093/mp/sss008]

Wang, Y., Zhang, X.B., Lu, S.J., et al., 2012. Inhibition of a basal transcription factor 3-like gene Osj10gBTF3 in rice results in significant plant miniaturization and typical pollen abortion. Plant Cell Physiol., 53(12):2073-2089. [doi:10.1093/pcp/pcs146]

Weaver, L.M., Lebrun, L., Franklin, A., et al., 1995. Molecular cloning of the biotinylated subunit of 3-methylcrotonylcoenzyme A carboxylase of Arabidopsis thaliana. Plant Physiol., 107(3):1013-1014. [doi:10.1104/pp.107.3.1013]

Wei, W., Ayad, N.G., Wan, Y., et al., 2004. Degradation of the SCF component Skp2 in cell-cycle phase G1 by the anaphase-promoting complex. Nature, 428(6979):194198. [doi:10.1038/nature02381]

Weil, R., Israel, A., 2004. T-cell-receptor and B-cell-receptormediated activation of NF- $\mathrm{kB}$ in lymphocytes. Curr. Opin. Immunol., 16(3):374-381. [doi:10.1016/j.coi.2004. 03.003]
Wen, R., Newton, L., Li, G., et al., 2006. Arabidopsis thaliana UBC13: implication of error-free DNA damage tolerance and Lys-63-linked polyubiquitylation in plants. Plant Mol. Biol., 61(1-2):241-253. [doi:10.1007/s11103-006-0007-x]

Wen, R., Torres-Acosta, J.A., Pastushok, L., et al., 2008. Arabidopsis UEV1D promotes Lysine-63-linked polyubiquitination and is involved in DNA damage response. Plant Cell, 20(1):213-227. [doi:10.1105/tpc.107.051862]

Wong, H.L., Sakamoto, T., Kawasaki, T., et al., 2004. Down-regulation of metallothionein, a reactive oxygen scavenger, by the small GTPase OsRac1 in rice. Plant Physiol., 135(3):1447-1456. [doi:10.1104/pp.103.036384]

Wu, X., Yamamoto, M., Akira, S., et al., 2009. Regulation of hematopoiesis by the K63-specific ubiquitin-conjugating enzyme Ubc13. PNAS, 106(49):20836-20841. [doi:10. 1073/pnas.0906547106]

Xiao, W., Lin, S.L., Broomfield, S., et al., 1998. The products of the yeast MMS2 and two human homologs (hMMS2 and CROC-1) define a structurally and functionally conserved Ubc-like protein family. Nucl. Acids Res., 26(17): 3908-3914. [doi:10.1093/nar/26.17.3908]

Yamaguchi, T., Kim, N.S., Sekine, S., et al., 1996. Cloning and expression of cDNA encoding a human ubiquitinconjugating enzyme similar to the Drosophila bendless gene product. J. Biochem., 120(3):494-497. [doi:10.1093/ oxfordjournals.jbchem.a021440]

Yamamoto, M., Sato, S., Saitoh, T., et al., 2006a. Cutting edge: pivotal function of Ubc13 in thymocyte TCR signaling. $J$. Immunol., 177(11):7520-7524. [doi:10.4049/jimmunol. 177.11.7520]

Yamamoto, M., Okamoto, T., Takeda, K., et al., 2006b. Key function for the Ubc13 E2 ubiquitin-conjugating enzyme in immune receptor signaling. Nat. Immunol., 7(9): 962-970. [doi:10.1038/ni1367]

Yang, R.F., Tang, Q.C., Wang, H.M., et al., 2011. Analyses of two rice (Oryza sativa) cyclin-dependent kinase inhibitors and effects of transgenic expression of OsiICK6 on plant growth and development. Ann. Bot., 107(7):10871101. [doi:10.1093/aob/mcr057]

Yang, Y., Liu, Z.H., Ware, C.F., et al., 1997. A cysteine protease inhibitor prevents activation-induced T-cell apoptosis and death of peripheral blood cells from human immunodeficiency virus-infected individuals by inhibiting upregulation of Fas ligand. Blood, 89(2):550-557.

Yang, Z., Wu, Y., Li, Y., et al., 2009. OsMT1a, a type 1 metallothionein, plays the pivotal role in zinc homeostasis and drought tolerance in rice. Plant Mol. Biol., 70(1-2): 219-229. [doi:10.1007/s11103-009-9466-1]

Yoshida, S., Forno, D.A., Cock, J.H., et al., 1976. Routine procedures for growing rice plants in culture solution. In: Laboratory Manual for Physiological Studies of Rice. IRRI, Los Baños, Philippines, p.61-66.

Yuan, J., Chen, D., Ren, Y., et al., 2008. Characteristic and expression analysis of a metallothionein gene, OsMT2b, down-regulated by cytokinin suggests functions in root development and seed embryo germination of rice. Plant 
Physiol., 146(4):1637-1650. [doi:10.1104/pp.107.110304]

Yuan, S., Fu, Y., Wang, X., et al., 2008. Voltage-dependent anion channel 1 is involved in endostatin-induced endothelial cell apoptosis. FASEB J., 22(8):2809-2820. [doi:10. 1096/fj.08-107417]
Zang, Y., Wang, Q., Xue, C., et al., 2012. Rice UBC13, a candidate housekeeping gene, is required for K63-linked polyubiquitination and tolerance to DNA damage. Rice, 5(1):24-34. [doi:10.1186/1939-8433-5-24]

\section{中文概要:}

本文题目：水稻泛素缀合酶基因 OsUbc13的分子特征和蛋白互作研究

Molecular identification and interaction assay of the gene (OsUbc13) encoding a ubiquitinconjugating enzyme in rice

研究目的: 通过研究水稻泛素缀合酶基因 $O s U b c 13$ 的序列特征、表达模式、亚细胞定位模式及其互作 分子, 为深入研究该基因的生物学功能和分子作用机理奠定基础。

创新要点: 首次对植物 Ubc13进行了亚细胞定位研究及蛋白互作研究。

研究方法: 通过序列比对及聚类分析进行 OsUbc13的序列特征研究; 通过实时荧光定量聚合酶链式反 应 (PCR) 进行 OsUbc13的表达模式分析; 通过聚乙二醇 (PEG) 介导转化烟草 BY-2 原生 质体进行 OsUbc13亚细胞定位研究（见图4）；通过酵母双杂交进行 OsUbc13的蛋白质互 作分析（见图5和表1）。

重要结论: OsUbc13编码具有 153 个氨基酸的蛋白质, 其推断的氨基酸序列与其它同源序列具有很高的 相似性; 该基因在水稻各组织中均有表达, 其中内稃、雌荵、雄荵和叶片中的表达量较高, 而根、茎和外稃中的表达量较低; 低温、甲基磺酸甲酯（MMS）和过氧化氢 $\left(\mathrm{H}_{2} \mathrm{O}_{2}\right)$ 胁 迫处理使胚性愈伤中 $O s U b c 13$ 的表达量显著上调, 甘露醇、脱落酸 ( $\mathrm{ABA}$ ) 和氯化钠 $(\mathrm{NaCl})$ 胁迫则使愈伤组织中该基因的表达量降低; OsUbc13 与绿色荧光蛋白（GFP）的融合蛋白 表达于质膜和核膜处; 酵母双杂交结果表明约有 20 个蛋白可能与 OsUbc13存在相互作用, 其中 OsVDAC（与细胞调亡有关）、OsMADS1（与花器官发育有关）、OsB22EL8（与活 性氧清除及 DNA 保护有关) 和 OsCROC-1（为 Lys63聚合泛素链形成及运行无误性 DNA 损伤耐受机制所必需) 四个蛋白经验证确与 OsUbc13互作。

关键词组: 水稻; 泛素缀合酶; 实时定量聚合酶链式反应; 酵母双杂交 